\section{LA DIDÁCTICA DE LA ARGUMENTACIÓN ESCRITA: UN RETO PARA AFRONTAR EN EL CONTEXTO UNIVERSITARIO}

Baaziz Saida

Para citar este artículo puede utilizar el siguiente formato:

Baaziz Saida: "La didáctica de la argumentación escrita: un reto para afrontar en el contexto universitario", Revista Atlante: Cuadernos de Educación y Desarrollo, ISSN: 1989-4155 (vol 13, № 7 octubre-diciembre 2021, pp. 40-52). En línea:

https://doi.org/10.51896/atlante/DZKM4733

\section{RESUMEN}

El objetivo de nuestra investigación consiste en realizar una aproximación a la didáctica de la argumentación escrita, considerándola como un eje vertebral que se aborda con mayor interés en la educación superior, ya que permite a los estudiantes transmitir y plasmar en forma escrita sus ideas, opiniones y pensamientos sustentados con criterios y argumentos válidos. Conviene señalar que esta tipología es compleja puesto que exige a los aprendientes la realización de razonamientos del pensamiento crítico. Por tal razón, hacemos hincapié a los diferentes estudios empíricos por medio de los cuales los estudiosos dieron a conocer las diferentes dificultades escriturarias a la hora de escribir un texto argumentativo. De igual modo, abordamos las secuencias didácticas destinadas a la mejora del proceso de redacción argumentativa, valiéndonos de unos estudios que dan luz y sugieren un camino a seguir con miras de enseñar la argumentación escrita de modo eficiente.

Palabras claves: Didáctica de Argumentación, texto argumentativo, dificultades escriturarias, habilidad argumentativa, secuencias didácticas.

\section{THE DIDACTICS OF WRITTEN: A CHALLENGE TO FACE IN THE UNIVERSITY CONTEXT}

\section{ABSTRACT}

The objective of our research is to make an approach to the didactics of written argumentation, considering it as a backbone that is addressed with greater interest in higher education, since it allows students to transmit and express in a written form their ideas, opinions and thoughts supported with valid criteria and arguments. It should be noted that this typology is complex since it requires the learners to carry out the reasoning of critical thinking. For this reason, we emphasize the different empirical studies through which scholars made known the different scriptural 
difficulties at the moment of writing an argumentative text. Similarly, we address the didactic sequences aimed at improving the argumentative writing process, using studies that shed light and suggest a path to follow with the aim of teaching written argumentation in an efficient way.

Keywords: Argumentation Didactics, argumentative text , scriptural difficulties, argumentative ability, didactic sequences.

\section{INTRODUCCIÓN}

Desde la antigüedad la difusión del saber se ha logrado a través de las habilidades de lectoescritura. De hecho, ambas destrezas son consideradas como recursos básicos para la formación académica de los estudiantes, cuya función es estimular el pensamiento crítico y desarrollar sus competencias intelectuales. Por lo tanto, estas dos habilidades cognitivas son trascendentales en el proceso de enseñanza y aprendizaje ya que propician apoyo para que los aprendientes comprendan y produzcan textos. En nuestro estudio, damos énfasis a la elaboración escrita mediante el tratamiento de la tipologia textual en el aula tales como; el texto narrativo, el descriptivo,el expositivo y el argumentativo. Al dar énfasis a la argumentación que se aborda con mayor énfasis en el contexto universitario, puesto que contribuye de manera eficaz en el desarrollo del pensamiento crítico del aprendiz, en la que ha de usar sus habilidades intelectuales para asumir una postura crítica respecto a un tema polémico.

En coherencia con lo anterior, la redacción argumentativa resulta una tarea compleja que requiere la realización de procesos de reflexión y de pensamiento crítico. De modo que, los estudiantes de la educación superior encuentran dificultades al elaborar textos argumentativos. Este hecho despertó el interés de varios didactas, que realizaron investigaciones por medio de las cuales dieron a conocer las dificultades de redacción de naturaleza argumentativa. En el nivel internacional, abordamos unos estudios del contexto Hispanoamericano, entre ellos se destacan trabajos de; Castro y Sánchez, (2013, 2018); Bañales, Vega, Reyna, Pérez y Rodríguez (2014, 2015); Camps, (1995); Sánchez y Álvarez, (1999), Perelman, (2001); Romano, (2020); Ramírez y Zamora, (2014). Etc. En el nivel nacional, referimos al contexto argelino, en el que aproximamos a las investigaciones de los docentes; Bellatreche (2011) y Belaouf (2016).

A la luz de los resultados obtenidos en los estudios anteriores por entidades nacionales e internacionales arrojaron resultados insatisfactorios e inquietantes por la baja calidad de las producciones argumentativas, lo que evidencia un rendimiento deficiente de la competencia textual argumentativa. Debido al desconocimiento y la falta de dominio de los componentes de la estructura argumentativa. En consonancia con lo enunciado, la complejidad de esta tipología y los resultados deficientes detectados nos motivaron en dar un panorama general de las secuencias didácticas para afrontar los retos que exige dicha habilidad escrita, basándonos en las más principales aportaciones de expertas y didactas de contextos ; Francés, Español y Latinoamericano.

\section{Aproximación a la didáctica de la argumentación escrita:}


La escritura es primordial en el ámbito educativo, ya que se ha establecido como una asignatura, a través de la cual se brinda a los estudiantes los elementos gramaticales y las estrategias de composición escrita. Se pretende desarrollar las destrezas comunicativas adecuadas con el fin de mejorar el quehacer académico-científico. Más bien, la escritura es una herramienta intelectual que permite al individuo de acceder a nuevas formas de conocer y de estructurar el pensamiento. Así como, está concebida como una actividad social porque permite la comunicación con otras personas sin límite de tiempo y espacio. Al respecto, afirma la profesora Camps "El uso de la lengua es una actividad compleja inscrita en el entramado social de prácticas comunicativorepresentativas. En este entramado el individuo construye su conocimiento, su pensamiento a partir de esta relación con los demás" (1997, p.1).

Cabe señalar que esta actividad escritural consiste en un proceso interactivo, comunicativo a través del cual el escritor narra hechos, describe fenómenos o situaciones, expone datos o conceptos específicos o bien argumenta una tesis sobre un tema polémico. Dentro de este enfoque de enseñar las diferentes formas textuales, en las que los aprendientes deben ajustarse a situaciones comunicativas reales de la vida social. En este ángulo, nos acercamos a las aseveraciones de Alvarado y Yeannoteguy (2000), afirman que la comunicación en todas sus esferas se realiza a través de los géneros discursivos, y los usuarios de la lengua incorporan en su comunicación un repertorio de géneros desde los más primarios del uso diario hasta los más complejos. De hecho, la práctica frecuente de estos géneros; periodísticos, académicos y religiosos proporcionan a los lectores y escritores ciertas habilidades que facilitan la lectura y la escritura de los diferentes tipos de textos con sus características lingüísticas; narrativos, descriptivos, explicativos y argumentativos .

En virtud de lo anterior, damos énfasis a la última tipología citada de naturaleza argumentativa que consiste en dar razones $u$ opiniones respecto a un asunto polémico y debatible que debe contener posturas a favor o en contra. En efecto, esta modalidad discursiva supone uno de los principales retos educativos al que se enfrentan los docentes y discentes en los actuales contextos de educación superior. De esta manera, los textos argumentativos adquieren mayor importancia en los currículos escolares cuyo objetivo consiste en influir en la opinión del lector para demostrarle que el punto de vista u opinión del autor es correcto o válido, Como lo afirman Bassols y Torrent " El hecho de argumentar es un signo distintivo del ser racional, es el arte de razonar a partir de opiniones generalmente aceptadas "(2012,p.31).

Por todo lo anterior, conviene aseverar que esta tipología textual permite al aprendiz de adquirir un sentido crítico de análisis, defender sus ideas y opiniones con fundamentos claros frente a múltiples situaciones que se presentan en su proceso de aprendizaje. En este ángulo, Castro y Sánchez (2018) señalaron que la carrera estudiantil universitaria es un desafío al que se debe enfrentar. Por tal objetivo de superar las dificultades y los obstáculos, se requiere saber reconstruir conocimientos a través de la participación activa en una variedad de tareas académicas; la lectura, la escritura y el pensamiento. Es decir, los aprendientes deberían realizar prácticas para mejorar su 
nivel y desarrollar sus habilidades de lectoescritura. De hecho, la lectura de textos especializados y el dominio de los mecanismos de la argumentación escrita para elaborar sus propios textos son de importancia capital, tanto en su formación superior, como en su práctica profesional. A este respecto, los autores adelantan "Plantear un problema, defender una tesis, evaluar un marco teórico o metodológico o construir una conclusión requieren por parte del hablante o del escritor habilidades de lenguaje y de pensamiento que se desarrollan de manera paulatina" (Castro y Sánchez, 2018, p.141).

\section{Esbozo panorámico de las dificultades escriturarias en el aula universitaria:}

Al dar un panorama de las diversas investigaciones llevadas a cabo en diferentes países, cuyo objetivo común es determinar las dificultades que encuentran los estudiantes en su proceso de redacción de textos argumentativos. Este tema, despertó el interés de varios estudiosos y pedagogos que realizaron intervenciones respecto a saber cuáles son los obstáculos que impiden a escribir una redacción argumentativa coherente y cohesionada. De ahí que, aproximamos a sus exhaustivos estudios, por medio de los cuales dieron aportes teóricos y hallazgos empíricos. En palabras generales, la habilidad de argumentación escrita trasciende las limitaciones geográficas, en nuestra investigación, no pretendemos tratar a los innumerables estudios al nivel internacional que hacen referencia a las dificultades escriturarias, pues nos limitamos en los contextos; Hispanoamericano y argelino.

\subsection{En el contexto Hispanoamericano:}

En el contexto Español se destaca el estudio de la catedrática Camps (1995) quien señaló que la contraargumentación representa mayor complejidad para los aprendices en los procesos de la comprensión y producción de textos. Por ello, propone la realización de tareas didácticas vinculadas a la habilidad de la argumentación escrita para promover la comprensión y superar las dificultades encontradas por los estudiantes a lo largo de su proceso composicional. Conviene resaltar que las dificultades que frenan los aprendientes son diversas tales como; la dificultad de entender un texto que involucra la intención del autor, las condiciones sociales del destinatario, además les resultó difícil determinar sus opiniones sobre diferentes temas, e incluso, plantearon la dificultad de entender las refutaciones, especialmente el uso de concesiones para defender la postura opuesta y la falta de manejo de los recursos lingüísticos.

En el contexto Mexicano, se destacan los trabajos de Castro y Sánchez (2013; 2018), Bañales, Vega, Reyna, Pérez y Rodríguez (2014; 2015). Estos autores, prueban que los estudiantes conllevan diferentes dificultades en su tarea de escritura argumentativa. Entre las cuales mencionaron; el desconocimiento de la función de las citas textuales, la imitación de la postura de las voces ajenas dando su apreciación personal como una extensión a la del experto para validar su discurso propio, la falta de manejo de la estructura argumentativa, confundir la argumentación cotidiana adquirida en la adolescencia y la argumentación académica, la falta de regular el proceso composicional mediante la realización de subprocesos de escritura; planificación, realización y 
revisión. Dado que, la razón principal de estas dificultades consiste en la enseñanza que reciben por docentes que aún son especialistas, pero no han sido formados en competencias para la enseñanza de escritura argumentativa.

De parecido modo, en el contexto de costarricense, se destaca la investigación de Ramírez y Zamora (2014) sobre la evaluación de la producción escrita de textos argumentativos producida por un grupo de la carrera de ingeniería industrial en el ámbito universitario. La tarea de la escritura argumentativa es percibida como compleja y difícil. De un lado, debido a la falta de entrenamiento de sus habilidades para argumentar sus opiniones en forma escrita. De otro lado, el desconocimiento del valor de dicha competencia que se usará posteriormente en el mundo profesional. En este estudio, se nota que los escritos analizados presentaban fallos y carencias por el desconocimiento de las estrategias necesarias para su construcción Por ello, resulta necesario formar y ejercitar sus habilidades a través de un modelo de enseñanza claro que ayude los aprendices a emprender el camino del aprendizaje.

En el contexto venezolano, citamos a los estudios empíricos llevados a cabo por Sánchez y Álvarez (1999), Serrano (2001), Serrano y Villalobos (2008). En síntesis, el análisis de los escritos producidos por los estudiantes arroja dificultades y carencias en su habilidad argumentativa. A causa del desconocimiento de la estructura argumentativa y la ausencia de los procedimientos discursivos para desarrollar sus ideas en forma escrita. De igual modo, en el contexto Chileno se destacan los estudios de Núñez (1999), Parodi y Núñez (1999), éstos estudiosos concuerdan en señalar la incapacidad de los aprendientes para construir un texto con una estructura mínima y dieron a conocer sus dificultados y errores respecto a los tres niveles prototípicos de la competencia textual.

En el contexto argentino, aludimos al trabajo de la psicopedagoga Perelman (2001) quien mencionó las dificultades que frenan al estudiante en el proceso de producción de un texto argumentativo tales como; la falta de la estructuración de su texto, el desconocimiento de las estrategias argumentativas para apoyar sus opiniones y la carencia en el uso de los recursos cohesivos. Más bien, la investigadora Romano (2020) afirmó que la razón de las dificultades de los estudiantes reside en confundir entre la argumentación cotidiana y académica. Por tal razón, insistió en la necesidad de reconstruir estrategias didácticas para ayudarles a adquirir la argumentación científica en cuanto a la densidad semántica del texto argumentativo, vocabulario, rigurosidad y objetividad.

\subsection{En el contexto argelino:}

En este renglón, se ubican dos valiosas investigaciones más sobresalientes y significativas al nivel nacional, ambas son de la Licenciatura del Francés. Hasta donde alcanza nuestro conocimiento podemos aseverar que esta destreza no ha sido tratada en el aula del español como Lengua Extranjera en el contexto universitario. Por tal razón, se nota casi la inexistencia de los estudios de la composición escrita en general, y en particular la tipología argumentativa, dado que la mayoría de los estudios realizados se enfatizaron en el estudio de la habilidad escrita de la enseñanza secundaria. 
El primer estudio realizado por el Doctor Bellatreche (2011), cuyo propósito, es mejorar el desempeño escrito de los estudiantes del primer año en el nuevo sistema de la licenciatura de Francés en la Universidad de Mostaganem, e incluso desarrollar sus habilidades de alfabetización, proponiendo procedimientos de recuperación y remediación para afrontar las dificultades encontradas en las copias de los sujetos. Es decir, realizó un análisis cuantitativo para evaluar sus habilidades respecto a tres aspectos; la organización de la macroestructura, que incluye; la introducción, el anuncio de plan y la conclusión, y la presencia de párrafos. El segundo aspecto, es el análisis de la argumentación que a su vez abarca criterios: como el uso de argumentos, ilustraciones, conectores.etc. El tercer nivel, se refiere a la extensión y la cantidad de la tasa de errores cometidos en el texto.

En virtud a lo anterior, los resultados del análisis de las redacciones argumentativas escritas por los informantes arrojaron resultados insatisfactorios. Primero, en el nivel de la macroestructura se indicó que los sujetos no demuestran habilidades sólidas en la organización de sus textos. En cuanto, el nivel de la lengua, el análisis evidencia que los estudiantes se enfocan en los errores ortográficos para evaluar su escritura, es decir, sus criterios de evaluación se limitan al nivel de la oración, ya que solo se fijan en los errores gramaticales al revisar sus textos. Finalmente, el nivel de las estrategias argumentativas, el análisis de sus copias muestra que son incapaces de respetar la organización macroestructural de sus textos.

En palabras generales, en la investigación se desprende que la mayoría de los estudiantes experimentan dificultades en su producción escrita con respecto a los tres aspectos textuales; la macroestructura, la calidad de la lengua y de la argumentación. Por tanto, desde la perspectiva pedagógica, el docente planteó una mejora de la enseñanza del texto argumentativo, a partir de las necesidades identificadas en los escritos analizados. De manera que, propuso una secuencia didáctica que aborda aspectos importantes para argumentar por escrito tales como; la comprensión del tema, el dominio de las habilidades de escritura argumentativa, la jerarquía de argumentos, la sustentación con ejemplos, el campo léxico relativo al tema en cuestión, la presencia de índices de enunciación y conectores.

En adición, nos aludimos a la investigación del doctor Belaouf (2016) quien centró su interés en la evaluación de las repercusiones de la implementación de una secuencia destinada a ayudar los estudiantes del segundo año de licenciatura Francesa del nuevo sistema LMD con el fin de mejorar su habilidad textual y, más particular, la construcción de textos argumentativos. El objetivo de su investigación consiste en proponer vías educativas que permitan a estos estudiantes remediar sus fallos a través de una enseñanza enfocada a la integración de la coherencia textual, asimismo poder determinar los avances logrados por los estudiantes al finalizar del curso. De modo que, el docente analizó el impacto de implementar estas secuencias en los escritos elaborados, en los que diagnosticó malformaciones y disfunciones que engloban tres etapas.

La primera fase vinculada a la destreza lingüística en la que los estudiantes muestran dificultades para utilizar el vocabulario, la sintaxis y los tiempos verbales, de hecho, la secuencia no 
tuvo un efecto positivo en el desarrollo de sus habilidades lingüísticas. Con respecto a la competencia de la planificación, los resultados indican que la mayoría tienen grandes deficiencias en la elaboración de un plan; introducción, cuerpo y conclusión. A sabiendas que, la secuencia proporcionada, aún no logre plenamente el objetivo establecido, pero tuvo un impacto considerable en la calidad de los textos producidos. Finalmente, la fase de la coherencia del texto, se puede concluir que los defectos aún persisten.

\section{Una encrucijada entre teoría y práctica de secuencias didácticas para la enseñanza de la argumentación escrita:}

En nuestro trabajo, hacemos hincapié, a los aportes de la didáctica de la argumentación escrita que ha sido una gran preocupación en el contexto Francés mediante las contribuciones de la escuela Ginebrina Dolz (1994, 1995), Dolz y Pasquier (1996, 2000), Cotteron (1995) cuyos trabajos han sido adaptados y traducidos al español. De igual modo, en el contexto español se destacan los trabajos de Camps (1995), Camps, A. y Dolz (1995), Camps y zayas (2006), Álvarez (2001), Ramírez (2006), Santamaría (1992). En América latina, sobresalen las propuestas de Díaz (2002), Serrano (2008), Martínez ( 2002), Rubio y Arias (2002), Ramírez (2010), Báñales y López (2016) etc. A continuación, abordamos algunas contribuciones didácticas de cada uno de los contextos citados.

\subsection{Aproximación teórica al concepto de la secuencia didáctica:}

Desde la perspectiva de la didáctica de la lengua, el concepto de Secuencia didáctica, en adelante (SD), ha sido desarrollado de manea amplia gracias a las propuestas teóricas y metodológicas de académicos de diferentes países. En nuestro trabajo, hacemos hincapié, a la investigación de la catedrática Camps (1995) desde el contexto español. De parecido modo, estimamos a los aportes de la didáctica del Fancés por las contribuciones de la escuela de Ginebra llevada a cabo por los estudiosos; Sánchez, Mosquera, Gagnon, Dolz (2012).

Al acercar a este término conviene citar a la investigación del estudioso Ramírez (2006) quien expuso un breve marco socioeducativo en el que se concreta el concepto en cuestión. De acuerdo con el autor, los planteamientos la secuencialidad se establece a través de la relación existida entre la escuela y la sociedad. En esta relación, se desprenden ejes centrales cuyos componentes son diversos. Por un lado, de un problema junto un asunto de estudio, y por otro lado, los participantes o sea los sujetos de enseñanza aprendizaje, es decir, el docente y el discente son agentes responsables en la explicitación de esta relación. Por lo tanto, la SD se parte de la dimensión socioeducativa en la que se plantean unos interrogantes tales como; el porqué, qué, a quien, cómo, cuándo enseñar y aprender. De hecho, el proceso educativo está orientado al planteamiento de problemas y la organización de soluciones alternativas con la finalidad de reconstruir la autonomía y realizar los fines educativos que éstos se llevan a cabo a través de los problemas y los conflictos específicos ubicados en contextos sociales e históricos.

A la luz de lo expuesto anteriormente, conviene agregar que los fundamentos didácticos y 
socioeducativos son bases que encaminan a la realización de la SD. Dado que, la vinculación entre ambos se lleva a cabo por vía de un hilo común determinado por un "tema", cuya función práctica consiste en combinarse el problema seleccionado como objeto y requisito de investigación para dirigir a la escuela hacia al entorno social, o bien guie al sujeto para que tenga una posición frente a los problemas sociales. De parecido modo, esta secuencia invita a los docentes a asumir su labor académica con mayor responsabilidad. En suma, la secuencia didáctica es un proceso que fomenta la participación activa de los estudiantes e involucra a los profesores en el desarrollo de un proyecto, que compone de unas cuestiones reales que asumen saberes dentro de la vida social y política. De modo que, su estructura admite la reflexión sobre el saber ser, hacer y el poder de los participantes en la toma de decisiones académicas, culturales y sociales

De acuerdo con el aporte de Camps (1995) quien propuso un modelo para enseñar la composición escrita, a partir de la necesidad en dar más valor a las actividades de escritura que se llevan a cabo en la escuela. Por tal objetivo, elaboró una serie de secuencias didácticas que sirven como eje que orientan la tarea de la redacción. La profesora afirma que la SD es una unidad de enseñanza de la composición escrita, que implica una serie de acciones organizadas y jerarquizadas por el docente, de acuerdo con los objetivos educativos. A sabiendas que, esta secuencia posee unos rasgos específicos; se elabora como un proyecto de trabajo cuya finalidad es producir un texto en un momento determinado,dado que su elaboración está introducido en una situación discursiva que le dará sentido.

De acuerdo con la contribución presentada por Sánchez y al (2012) en la universidad de Ginebra, Suiza, abordaron la SD en Ele como recurso didáctico, enfatizando en la elaboración de secuencias didácticas para la enseñanza de los géneros textuales que han sido basados en cuatro fases. En la primera fase, se ejerce la producción inicial para identificar las potencialidades y dificultades de los alumnos. En la segunda etapa, mediante el análisis de las producciones, se planifican contenidos de la secuencia. En la tercera instancia, se combinan las actividades con miras de producir un género en el aula, que deben ser jerarquizadas en módulos que plantean de manera concreta los aspectos vinculados al género textual en cuestión. La última fase, es la producción final concebida como una reformulación de la primera producción, o bien la escritura de un nuevo texto.

\subsection{Hacia la propuesta de secuencias didácticas:}

Empezándonos en el tratamiento de la argumentación escrita en la escuela ginebrina. De una parte, nos remitimos a las aportaciones de Dolz (1994) propuso un estudio sobre las posibilidades de la articulación entre las actividades de interacción oral; diálogo, discusión, debate, monólogo y de situación de producción argumentativa. Considerando que el desarrollo de la argumentación oral se considera la condición fundamental para abordar la argumentación escrita. Por ello, discutió la relación entre ambas modalidades desde la perspectiva lingüística y didáctica. En al año posterior (1995) trató brevemente la interacción de la lectoescritura, de modo que reveló cómo incide la escritura en la mejora de la comprensión lectora, e incluso brindó una serie de seis elementos básicos para enseñar la argumentación; indagando su situación, la estructura de base de los 
argumentos, sus operaciones específicas, las estrategias y los procedimientos retóricos, las unidades lingüísticas y la planificación.

De otra parte, se destacan los trabajos realizados por Dolz y Pasquier $(1996,2000)$, partieron de una propuesta basada en la enseñanza de la expresión oral y escrita de un modo sistémico y eficiente. Esta sugerencia didáctica sirvió como una ilustración del método de trabajo que ha sido adoptada en diversos países. Conviene resaltar que su modelo de secuencias didácticas destinado a satisfacer las necesidades de los docentes y de los discentes. De modo que, cada secuencia se fundamenta a partir de un género textual o un tipo de discurso. En síntesis, toda SD requiere "La constitución de un corpus textual auténtico: documentos escritos y orales relativos a un género textual que son tanto la referencia para organizar actividades y ejercicios como el modelo de inspiración para los alumnos" (Dolz, y Pasquier, 1996, p.7).

Acorde a los autores abogan que las secuencias fueron elaboradas tomando en cuenta las dificultades a las que enfrentan los aprendientes al argumentar. Por consiguiente, propusieron una gama de estrategias para solventar los problemas hallados mediante de la variación de actividades de tipo colaborativo o individual, asimismo de los ejercicios de preparación para dar la oportunidad a cada aprendiente de acceder a distintos nociones y competencias, y de renovar los métodos del trabajo. En definitiva, la estructura básica de las SD, es organizada en tres fases: desde la producción inicial con el fin de observar las habilidades y dificultades del grupo, luego el desarrollo de módulos o talleres de aprendizaje sobre las dimensiones textuales problemáticas. Por último, la producción final de textos orales o escritos para brindar al estudiante la posibilidad de ejercer los conocimientos abordados.

En la misma línea, se destaca la investigación de Santamaría (1992), mediante la cual experimentó una secuencia para enseñar la composición argumentativa, surgida frente a las diversas necesidades halladas en los alumnos como; los errores frecuentes ortográficos, la falta de conexión en las oraciones, las confusiones en la puntuación, la incoherencia, la falta de creatividad y las carencias en el vocabulario y en la estructuración lógica del discurso. Por ello, propuso una SD que contiene cuatro etapas; actuar, reflexionar, analizar y generalizar. Cada fase partió de diversas sugerencias didácticas en las que las actividades se diseñan de acuerdo con los planteamientos de cada fase. Al explicar las etapas, conviene resaltar que la primera y la cuarta se denominan; actuación y generalización inspirándose en los enfoques didácticos basados en las funciones y en el proceso propuestos por Cassany (1999), en cuanto la segunda se llama; reflexión basada en el contenido, y la tercera cuya denominación es Análisis tomando como referencia el enfoque basado en la gramática.

De acuerdo con Camps (1995) propuso una SD para la enseñanza y aprendizaje de la argumentación escrita que conlleva once sesiones didácticas, partiendo de una fase inicial en la que los alumnos deben escribir un texto respecto a una consigna dada, y luego la realización de una serie de actividades orientadas a comprender las estructuras contraargumentativas que abarcan ; la concesión y la refutación mediante la lectura y el análisis de los textos y la realización debates para 
contrastar los opiniones sobre un asunto determinado, en la fase final los sujetos han de escribir un texto que comparte los mismos criterios del texto inicial. En el mismo año realizó otro estudio, junto con Dolz (1995) asumieron un trabajo sobre la argumentación y su objetivo en la enseñanza, abordando su trascendencia en la educación del ciudadano y en su incidencia en el aprendizaje de la expresión oral y escrita, e incluso reivindican un decálogo para argumentar en diferentes situaciones.

Dentro del mismo hilo de ideas, nos remitimos a la investigación de Ramírez (2006) sobre el proceso de redacción de textos. Acorde con sus planteamientos, la SD recurre al tratamiento de diversos problemas de tipo social, académico y estético concebidos como una estrategia para promover la creatividad de acuerdo con los intereses educativos, e incluso se permite la evaluación de las metas propuestas. En resumen, la SD no procura a dar remedios definitivos a los problemas escriturales o de cualquier otra competencia, más bien pretende mejorar las prácticas docentes en el aula dentro de una dimensión sociocultural. Por su parte, propuso una secuencia para potenciar la capacidad argumentativa de los aprendientes, valiéndose en la elaboración de una carta petitoria, desarrollada en ocho sesiones con una doble finalidad; conseguir un objetivo discursivo en la que el estudiante interactúa en un discurso de recepción real, y un objetivo de aprendizaje que incide en el proceso de elaboración de los textos escritos.

Terminamos con el estudio de los autores Báñales y Vega (2016) en el que propusieron enseñar la habilidad de la argumentación escrita en el entorno universitario.De igual modo, revelaron una serie de decisiones pedagógicas, junto con la descripción de unas secuencia didácticas. Con miras a facilitar la tarea docente, considerándola como una competencia importante. En el lado personal, se convierte en una forma de razonamiento para expresar opiniones sobre temas polémicos. En el lado académico, es un recurso didáctico para aprender y acumular conocimientos. Por ello, en los últimos años ha atendido un amplio abanico de necesidades formativas en los contextos de la educación superior; anglosajones, iberoamericanos y latinoamericanos.

\section{CONCLUSIONES}

En sintonía con lo anterior, la argumentación escrita se considera una herramienta poderosa para desarrollar las habilidades del pensamiento crítico de los aprendientes en la educación superior. En síntesis, es una habilidad permite a los estudiantes expresar sus ideas, opiniones, dando sus posturas personales, a favor 0 en contra respecto a temas polémicos que suscitan un debate y sustentarlas con argumentos válidos. En este sentido, el proceso argumentativo escritural pasa a ser uno de los retos en el contexto universitario. Por ende, radica una importancia crucial en enseñar dicha habilidad correctamente, incorporando estrategias didácticas para facilitar su aprendizaje.

En el presente artículo, hemos revisado distintos estudios llevados a cabo en muchos países que revelan las dificultades a la hora de redactar un texto argumentativo. Por ende, la mayoría de los análisis de las producciones arrojan resultados muy descendidos e insatisfactorios. Los problemas, son distintos entre ellos se destacan; el planteamiento de la tesis, la falta de presentación de argumentos rigurosos, sus escritos no presentan una estructura textual definida. A partir de los 
resultados obtenidos los didactas insistieron en la necesidad de enfrentar los obstáculos que frenan el proceso de redacción por vía de las propuestas de secuencias didácticas para guiar el estudiante en el proceso de la escritura argumentativa.

\section{REFERENCIAS}

Alvarado, M. y Yeannoteguy, A. (2000). La escritura y sus formas discursivas. Curso introductorio. Editorial Universitaria de Buenos Aires: Eudeba.

Álvarez, T. (2001). Textos expositivos-explicativos y argumentativos. Barcelona: Octaedro.

Bañales, G. y Vega, N. (2016). La enseñanza estratégica de la argumentación escrita en las disciplinas: desafíos para los docentes universitarios .En G. Bañales, M. Castelló y N. Vega (Coords.), Enseñar a Leer y Escribir en la Educación Superior. Propuestas educativas basadas en la invesfigación, (pp.101-127). México, D.R.

Bañales, G., Vega, N., Araujo, N., Reyna, A. y Rodríguez, B. (2015). "La enseñanza de la argumentación escrita en la universidad. Una experiencia de intervención con estudiantes de lingüística aplicada ". Revista Mexicana de Investigación Educativa, 20(66), pp. 879-910.

Bañales, G., Vega, A.; Reyna, A., Pérez, E. y Rodríguez, B. (2014). "La argumentación escrita en las disciplinas: retos de alfabetización de los estudiantes universitarios". Revista Internacional de Ciencias Sociales y Humanidades, XXIV, 2, pp. 29-52.

Bassols, M. y A. Torrent. (2012). Modelos textuales. Barcelona :Octaedro.

Belaouf, M. (2016). L'appropriation de l'écrit argumentatif en contexte universitaire à travers la notion de cohérence. [Thèse doctoral. Université de Mostaganem ].

Bellatrèche, H. (2013), Apprendre la production écrite en première année de licence de français nouveau régime en Algérie : entre le dire et le faire. Le cas du texte argumentatif. [Thèse de doctorat, Université de Mostaganem ].

Camps, A. (1995). "Aprender a escribir textos argumentativos: Características dialógicas de la argumentación escrita". Infancia y aprendizaje (25), pp. 51-63.

Camps, A. (1997). "Escribir. La enseñanza y el aprendizaje de la composición escrita. En revista Signos. Teoría y práctica de la educación". (20), pp. 24-33.

Camps, A y Dolz , J. (1995). "Introducción: enseñar a argumentar: un desafío para la escuela actual". Comunicación, lenguaje y educación, año: 07, n .25, pp. 5-8.

Camps, A y Zayas, F y (coord.) (2006). Secuencias didácticas para aprender gramática. Barcelona : Graó.

Cassany, D. (1999). Construir la escritura, Barcelona :Paidós

Castro, M.C. y Sánchez, M.( 2013). "La expresión de opinión en textos académicos escritos por estudiantes universitarios". Revista mexicana de investigación educativa, 18(57),pp. 483-506.

Castro, M.C. y Sánchez, M. (2018). La construcción de opinión: posicionamiento y voz en textos académicos. En María Cristina Castro Azuara y al. . La lectura y la escritura en las disciplinas: lineamientos para su enseñanza (pp.137-166). Buenos Aires, Argentina: Los Polvorines.

Cotteron, J. (1995). "¿Secuencias didácticas para enseñar a argumentar en la primaria?" Comunicación, Lenguaje y Educación, (7),pp. 79-94. 
Díaz, A. (2002). La argumentación escrita. Medellín, Colombia: Universidad de Antioquia.

Dolz, J. y Pasquier, A. (1996). Argumentar para convencer. Una secuencia didáctica de iniciación al texto argumentativo para el primer ciclo de la Educación Secundaria Obligatoria. Traducción de Pilar Labaín, María José Sánchez y Francisco Sierra. Gobierno de Navarra, Departamento de Educación y Cultura.

Dolz, J. y Pasquier, A. (2000). Escribo mi opinión. Una secuencia didáctica de iniciación a los textos de opinión para el tercer ciclo de educación primaria. España: Gobierno de Navarra, Departamento de Educación y Cultura.

Dolz, J. (1994). "La interacción de las actividades orales y escritas y la enseñanza de la argumentación". Comunicación, Lenguaje y Educación, 23, pp. 17-27.

Dolz, J. (1995). "Escribir textos argumentativos para mejorar su comprensión". En A. Camps \& J. Dolz (Coord.), Comunicación, Lenguaje y Educación. Enseñar a argumentar, pp. 65-77. Madrid: Edisa.

Martínez Solís, M. (2002). Estrategias de lectura y escritura de textos: Perspectivas teóricas y talleres. Cali : Universidad del Valle.

Martínez Solís, M. (2002). Propuesta de intervención pedagógica para la comprensión y producción de textos académicos. Cátedra UNESCO. Cali : Universidad del Valle

Núñez, L. P. (1999). "La construcción de textos argumentativos escritos en los inicios de la adolescencia de jóvenes chilenos ". En G. Parodi (ed.), Discurso, cognición y educación, pp. 159-178. Chile: Ediciones Universitarias de Valparaíso.

Parodi, S.G. y P. Núñez.(1999). "La búsqueda de un modelo cognitivo/textual para la evaluación del texto escrito". En M. Martínez Solís (ed.) (1999) Comprensión y producción de textos académicos. Cátedra UNESCO, Universidad del Valle.

Perelman, F. (2001). "Textos argumentativos: su producción en el aula ". En lectura y vida Revista latinoamericana de lectura .Año XXII, NUM .2, JUNIO .Buenos Aires. Asociación Internacional de lectura, pp. 32-45.

Ramírez, R .(2010). Didácticas de la lengua y de la argumentación escrita. Editorial Universitaria Universidad de Nariño: Pasto.

Ramírez, J.A., Zamora, J.R. (2014). "La producción escrita de textos argumentativos en la educación superior” . Revista de Lenguas Modernas,(20), pp. 167-181.

Ramírez, R. (2006). "Secuencias didácticas en los procesos de enseñanza-aprendizaje de la competencia argumentativa escrita". Folios, 24,pp. 27-43.

Romano, M. B. (2020). “ Argumentar en la universidad: Representaciones estudiantiles, retos y perspectivas”. Revista Iberoamericana De Argumentación, (20), 206-227.

Rubio, M., y Arias V. (2002). " Una secuencia didáctica para la enseñanza de la argumentación escrita en el Tercer Ciclo". Lectura y vida, 23(4), pp. 34-41

Sánchez, I. y N. Álvarez (1999). "El discurso argumentativo de los escolares venezolanos: ¿evolución o estancamiento?". Ponencia presentada en el III Coloquio Internacional de Análisis del Discurso. Santiago, Chile, pp.1-10 .

Sánchez Abchi, V., Mosquera, S., Gagnon, R. y Dolz, J. (2012). "La secuencia didáctica en ELE: una 
herramienta para la enseñanza y un dispositivo para la formación docente". Boletín Helvético Hispánico 19 ,pp. 195-221.

Santamaria, J. (1992). "Escribir textos argumentativos: una secuencia didáctica ", en: Camps, Anna: Secuencias didácticas para aprender a escribir. Barcelona: Grao, 2003,pp. 111-126.

Serrano, S. (2001). "La argumentación como problema en la composición escrita de estudiantes de formación docente". Lectura y Vida, 22 (4), pp. 26-37.

Serrano, S. (2008). "Composición de textos argumentativos. Una aproximación didáctica". Revista de Ciencias Sociales (Ve), XIV (1) ,pp.149-161.

Serrano, S y Villalobos, J. (2008). "Niveles discursivos del texto argumentativo escrito por estudiantes de formación docente”. .Universidad de Los andes: Mérida, pp . 152-187. 